\title{
Robust Transceiver Design for MIMO System with Amplify-and-Forward Relay
}

\author{
Jun-Xu Su, Xiao-Min Chen, Qiu-Ming Zhu, Yi-Min Zhu, Zhu Fang
}

\begin{abstract}
In this paper, a robust transceiver design for dual-hop multiple-input multiple-output (MIMO) system with amplify-and-forward (AF) relay is produced in the presence of imperfect channel state information (CSI) . The antenna correlation at both ends of the channel and the channel estimation errors are taken into account. The optimization problem is formulated based on minimum mean-square error (MMSE) rule, and the original problem is decoupled into three convex sub-problems. Then the source precoding matrix is optimized by the interior-point method, the optimal relaying matrix is derived by lagrangian multiplier method. The equalizer is optimized by a gradient-based line search algorithm and an alternating algorithm is proposed for the joint design. Simulation results indicated that the proposed design approach achieves better robustness against antenna correlation and channel estimation errors than other approaches.
\end{abstract}

Index Terms - MIMO relay system, amplify-and-forward, mean-square error (MMSE), antenna correlation, channel estimation errors.

\section{INTRODUCTION}

In the past decade, relay-assisted cooperative transmission as a promising technique to improve the system reliability and extend the network coverage has gained much interest from both academic and industrial communities [1]. Multiple-input multiple-output (MIMO) technique is known as a remarkable breakthrough in wireless communications that could bring substantial diversity and multiplexing gains [2]. The combination of MIMO and relay technique could take the advantages of both techniques, and has been considered as an important candidate for future wireless networks [3].

Some existing studies have investigated for MIMO relay systems. The optimal transceiver based on minimum mean-square error (MMSE) criterion are investigated in [3]. Several joint LMMSE transceivers with perfect CSI have been investigated to improve the system BER performance further in [4]-[6]. However, all these works assume that channel state information (CSI) is perfectly known. Unfortunately, this assumption cannot be met in practice due to various reasons, because antenna correlation and the channel estimation errors are always inevitably exist. Some joint precoding schemes are discussed with considering channel estimation error in [7], [8]. Robust transceiver

\footnotetext{
Manuscript received April 16, 2016; revised June 17, 2016. This work is supported by the Fundamental Research Funds for the Central Universities.( NO.NS2015046,NO.NS2016044).

The authors are with the College of Electronic and Information Engineering, Nanjing University of Aeronautics and Astronautics, Nanjing, China (e-mail: chenxm402@nuaa.edu.cn).
}

designs based on antenna correlation are introduced in [9], [10], but the CSI is supposed to be perfect estimated.To the best of our knowledge, in the existing works less robust transceiver design in MIMO relay systems has been obtained in the presence of channel correlation and estimation errors.

In this paper, a robust transceiver for dual-hop MIMO system with AF relay is investigated where antenna correlation and the channel estimation errors are taken into account .The rest of the paper is organized as follows. In Section II, the system model and channel model are presented. In Section III, a MMSE-based optimization problem is formulated. the source precoding matrix is optimized by the interior-point method, the optimal relaying matrix is derived by lagrangian multiplier method, the equalizer is optimized by a gradient-based line search algorithms and an alternating algorithm is proposed for the joint design are given in Section IV. Section V provides simulation results, and Section VI concludes the paper.

Notations: Boldface uppercase and lowercase letters denote matrices and vectors; $(\bullet)^{\mathrm{T}},(\bullet)^{*},(\bullet)^{\mathrm{H}},(\bullet)^{-1},(\bullet)^{+}, \varepsilon(\bullet)$ and $\operatorname{tr}(\bullet)$ denotes the transpose, conjugate, conjugate transpose, inverse, Moore-Penrose pseudo-inverse, expectation and trace of a matrix, respectively; $\operatorname{diag}(\boldsymbol{X})$ is a diagonal matrix formed form elements of the main diagonal of $\boldsymbol{X} ; \boldsymbol{X}^{1 / 2}$ is the square root of a positive semi-definite matrix $\boldsymbol{X}, \boldsymbol{\lambda}_{\max }(\boldsymbol{X})$ denotes the maximum eigenvalue of matrix.

\section{SyStem MOdEL AND CHANNEL MODEL}

\section{A. System Model}

A dual-hop Amplify-and-Forward MIMO relay system model [3] is shown in Fig. 1. The source node transmits information to the destination node with the help of a relay station, where $n_{\mathrm{s}}$ denotes the antenna number of source node. The relay station and the destination node have $n_{\mathrm{r}}$ and $n_{\mathrm{d}}$ antennas, respectively. In order to support $n_{\mathrm{s}}$ independent data streams simultaneously, there should be satisfied with $n_{\mathrm{s}} \leq \min \left(n_{\mathrm{r}}, n_{\mathrm{d}}\right)$. It is also assumed that the relay station operates in half-duplex mode and the transmission takes place in two consecutive time slots.

In the first time slot, source node transmits its information $x$ to the relay station simultaneously. Let $\boldsymbol{x}=\left[\boldsymbol{x}_{1}^{T}, \ldots, \boldsymbol{x}_{n_{s}}^{T}\right]^{T} \in \mathbb{C}^{n_{s} \times 1}, \varepsilon\left[\boldsymbol{x} \boldsymbol{x}^{H}\right]=\boldsymbol{I}_{n_{s}}$, then the received signal vector $\mathbf{y}_{\mathrm{r}} \in \mathbb{C}^{n_{r} \times 1}$ at the relay station can be 
expressed as

$$
\boldsymbol{y}_{\mathrm{r}}=\boldsymbol{H B x}+\boldsymbol{w}
$$

where $\boldsymbol{B} \in \mathbb{C}^{n_{s} \times n_{s}}$ denotes the precoding matrix, $\operatorname{tr}\left(\boldsymbol{B} \boldsymbol{x} \boldsymbol{x}^{H} \boldsymbol{B}^{H}\right)=\operatorname{tr}\left(\boldsymbol{B} \boldsymbol{B}^{H}\right) \leq P_{s}$, and $P_{s}$ is the maximum transmission power at the transmitter. $\boldsymbol{H} \in \mathbb{C}^{n_{r} \times n_{s}}$ is the MIMO channel matrix between the transmitter and the relay station, and $\boldsymbol{w}$ is the additive white Gaussian noise (AWGN) vector with zero mean and covariance matrix $\boldsymbol{R}_{w}=\varepsilon\left[\boldsymbol{w} \boldsymbol{w}^{H}\right]=\sigma_{w}^{2} \boldsymbol{I}_{n_{r}}, \sigma_{w}^{2}$ is the variance of $\boldsymbol{w}$.

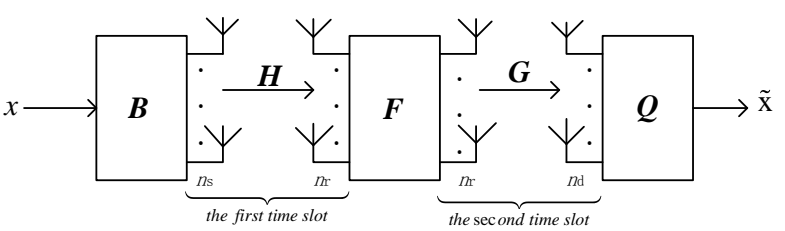

Fig. 1. Block diagram of MIMO relay system.

In the second time slot, the relay station multiples its received signal by a linear processing matrix $\boldsymbol{F} \in \mathbb{C}^{n_{r} \times n_{r}}$ and forwards it to the destination node, hence the transmitted signal vector $\boldsymbol{Y} \in \mathbb{C}^{n_{r} \times 1}$ at the relay station can be expressed as

$$
\boldsymbol{Y}=\boldsymbol{F} \boldsymbol{y}_{r}
$$

where the transmission power satisfies $\operatorname{tr}\left(\boldsymbol{Y} \boldsymbol{Y}^{H}\right) \leq P_{r}, P_{r}$ is the maximum transmission power at the relay station.

Let $\boldsymbol{G} \in \mathbb{C}^{n_{d} \times n_{r}}$ be the channel matrix from the relay station to the destination node, then the received signal vector $r \in \mathbb{C}^{n_{d} \times 1}$ is

$$
\boldsymbol{r}=\boldsymbol{G Y}+\boldsymbol{n}=\boldsymbol{G F H S}+\boldsymbol{G F w}+\boldsymbol{n}
$$

where $\boldsymbol{n} \in \mathbb{C}^{n_{d} \times 1}$ is the AWGN vector at the destination node with zero mean and covariance matrix $\boldsymbol{R}_{n}=\varepsilon\left[\boldsymbol{n n} \boldsymbol{n}^{H}\right]=\sigma_{n}^{2} \boldsymbol{I}_{n_{d}}, \sigma_{n}^{2}$ is the variance of $\boldsymbol{n}$.

The linear receiver at the destination is adopted to retrieve the transmitted signal vector of the source node. Denote the linear receiver matrix as $\boldsymbol{Q} \in \mathbb{C}^{n_{s} \times n_{d}}$, then the estimated signal vector $\hat{\boldsymbol{x}} \in \mathbb{C}^{n_{s} \times 1}$ is given by

$$
\begin{aligned}
\hat{\boldsymbol{x}} & =\boldsymbol{Q} \boldsymbol{r}=\boldsymbol{Q G F H S}+\boldsymbol{Q G F w}+\boldsymbol{Q n} \\
& =Q G F H B a+Q G F w+Q n
\end{aligned}
$$

\section{B. Channel Model}

The Kronecker model ${ }^{[11]}$ is used to describe the antenna correlation of $\boldsymbol{H}$ and $\boldsymbol{G}$ as $\boldsymbol{H}=\Sigma_{H}^{1 / 2} \tilde{\boldsymbol{H}} \boldsymbol{\Psi}_{H}^{1 / 2}$ and $\boldsymbol{G}=\Sigma_{G}^{1 / 2} \tilde{\boldsymbol{G}} \boldsymbol{\Psi}_{G}^{1 / 2}$, where $\boldsymbol{\Psi}_{H}, \boldsymbol{\Psi}_{G}$ are the transmit correlation matrix, and $\Sigma_{H}, \Sigma_{G}$ are the receive correlation matrices for $\boldsymbol{H}$ and $\boldsymbol{G}$, respectively. The correlation matrices are positive semi-definite and perfectly known. The entries of $\tilde{\boldsymbol{H}}$ and $\tilde{\boldsymbol{G}}$ are independent and identically distributed (i.i.d.) Gaussian random variables with zero-mean and unit variance. However, in practical networks, it is hard to obtain the perfect CSI. Define $\tilde{\boldsymbol{H}}=\hat{\boldsymbol{H}}+\Delta \boldsymbol{H}$ and $\tilde{\boldsymbol{G}}=\hat{\boldsymbol{G}}+\Delta \boldsymbol{G}$, where $\hat{\boldsymbol{H}}$ and $\hat{\boldsymbol{G}}$ are the estimation of $\tilde{\boldsymbol{H}}$ and $\tilde{\boldsymbol{G}}$, and $\Delta \boldsymbol{H}, \Delta \boldsymbol{G}$ are the estimation errors matrices whose elements are i.i.d. zero-mean Gaussian random variables with variances $\sigma_{H}^{2}$ and $\sigma_{G}^{2}$, respectively. Hence, the channels can be further expressed as

$$
\begin{aligned}
& \boldsymbol{H}=\Sigma_{H}^{1 / 2}(\hat{\boldsymbol{H}}+\Delta \boldsymbol{H}) \boldsymbol{\Psi}_{H}^{1 / 2}=\overline{\boldsymbol{H}}+\Sigma_{H}^{1 / 2} \Delta \boldsymbol{H} \boldsymbol{\Psi}_{H}^{1 / 2} \\
& \boldsymbol{G}=\Sigma_{G}^{1 / 2}(\hat{\boldsymbol{G}}+\Delta \boldsymbol{G}) \boldsymbol{\Psi}_{G}^{1 / 2}=\overline{\boldsymbol{G}}+\Sigma_{G}^{1 / 2} \Delta \boldsymbol{G} \boldsymbol{\Psi}_{G}^{1 / 2}
\end{aligned}
$$

\section{THE MMSE-BASED TRANSCEIVER DESIGN PROBLEM FORMULATION}

In this section, the MSE of the received signal at the destination is derived in the presence of channel correlation and estimation errors. The MMSE-based transceiver design problem will be formulated with the constraint of maximum transmission power at the relay and the transmitter.

According to the system model and channel model, the MSE of the estimated signal at the destination can be expressed as

$$
\begin{array}{rl}
M S & E(\boldsymbol{B}, \boldsymbol{F}, \boldsymbol{Q})=\varepsilon\left(|\hat{\boldsymbol{x}}-\boldsymbol{x}|^{2}\right) \\
= & \varepsilon\left(|\boldsymbol{Q} \boldsymbol{G} \boldsymbol{F} \boldsymbol{H} \boldsymbol{x}+\boldsymbol{Q G} \boldsymbol{F} \boldsymbol{w}+\boldsymbol{Q n}-\boldsymbol{x}|^{2}\right) \\
= & \varepsilon\left(\mid \boldsymbol{Q}\left(\overline{\boldsymbol{G}}+\Sigma_{G}^{1 / 2} \Delta \boldsymbol{G} \boldsymbol{\Psi}_{G}^{1 / 2}\right) \boldsymbol{F}\left(\overline{\boldsymbol{H}}+\Sigma_{H}^{1 / 2} \Delta \boldsymbol{H} \boldsymbol{\Psi}_{H}^{1 / 2}\right) \boldsymbol{B} \boldsymbol{x}\right. \\
& \left.+\boldsymbol{Q}\left(\overline{\boldsymbol{G}}+\Sigma_{G}^{1 / 2} \Delta \boldsymbol{G} \boldsymbol{\Psi}_{G}^{1 / 2}\right) \boldsymbol{F} \boldsymbol{w}+\boldsymbol{Q n}-\left.\boldsymbol{x}\right|^{2}\right) \\
= & \operatorname{tr}\left[\boldsymbol{Q} \overline{\boldsymbol{G}} \boldsymbol{F} \boldsymbol{Z} \boldsymbol{F}^{H} \overline{\boldsymbol{G}}^{H} \boldsymbol{Q}^{H}+\boldsymbol{I}_{n_{s}}+\boldsymbol{Q} \boldsymbol{R}_{n} \boldsymbol{Q}^{H}\right] \\
& +\operatorname{tr}\left[\sigma_{G}^{2} \operatorname{tr}\left(\boldsymbol{F} \boldsymbol{Z} \boldsymbol{F}^{H} \boldsymbol{\Psi}_{G}\right) \boldsymbol{Q} \boldsymbol{\Sigma}_{G} \boldsymbol{Q}^{H}\right] \\
& -\operatorname{tr}\left(\boldsymbol{Q} \overline{\boldsymbol{G}} \boldsymbol{F} \overline{\boldsymbol{H}} \boldsymbol{B}+\boldsymbol{B}^{H} \overline{\boldsymbol{H}}^{H} \boldsymbol{F}^{H} \overline{\boldsymbol{G}}^{H} \boldsymbol{Q}^{H}\right)
\end{array}
$$

Define $\boldsymbol{Z}=\left[\overline{\boldsymbol{H}} \boldsymbol{B} \boldsymbol{B}^{H} \overline{\boldsymbol{H}}^{H}+\sigma_{H}^{2} \operatorname{tr}\left(\boldsymbol{B} \boldsymbol{B}^{H} \boldsymbol{\Psi}_{H}\right) \boldsymbol{\Sigma}_{H}+\boldsymbol{R}_{w}\right]$, the power constraint at the transmitter and the relay station can be rewritten as

$$
\begin{gathered}
\operatorname{tr}\left(\boldsymbol{B} \boldsymbol{B}^{H}\right) \leq P_{s} \\
\operatorname{tr}\left(\boldsymbol{Y} \boldsymbol{Y}^{H}\right)=\operatorname{tr}\left[\boldsymbol{F}\left(\boldsymbol{H B B} \boldsymbol{B}^{H} \boldsymbol{H}^{H}+\boldsymbol{R}_{w}\right) \boldsymbol{F}^{H}\right] \\
=\operatorname{tr}\left[\boldsymbol{F} \boldsymbol{Z} \boldsymbol{F}^{H}\right] \leq P_{r}
\end{gathered}
$$

With the transmit power constraint, the sum MSE minimization problem can be formulated as

$$
\begin{aligned}
& \arg \min _{\boldsymbol{B}, \boldsymbol{F}, \boldsymbol{Q}}\{M S E\} \\
& \text { s.t } \operatorname{tr}\left(\boldsymbol{B} \boldsymbol{B}^{H}\right) \leq P_{s} \\
& \operatorname{tr}\left[\boldsymbol{F} \mathbf{Z} \boldsymbol{F}^{H}\right] \leq P_{r}
\end{aligned}
$$

\section{MMSE-BASED TRANSCEIVER DESIGN}

It is clear that the problem (10) is non-convex with respect to $\boldsymbol{B}, \boldsymbol{F}$ and $\boldsymbol{Q}$ jointly, and is hard to solve analytically. Nevertheless, it can be verified that the problem is convex in each variable when the others are fixed. Hence, the original problem can be decoupled into three convex sub-problems. Afterwards, an iterative algorithm is proposed to jointly design the transceiver.

\section{A. Optimization of Precoding Matrix $\boldsymbol{B}$}

The sub-problem to optimize $\boldsymbol{B}$ for fixed $\boldsymbol{F}$ and $\boldsymbol{Q}$ can be 
written as

$$
\begin{aligned}
\mathrm{mi}_{\boldsymbol{B}} & \boldsymbol{b}^{H} \boldsymbol{A}_{1} \boldsymbol{b}-\otimes R \boldsymbol{C}_{l}^{H} \boldsymbol{b}+D_{1} \\
\text { s.t } & \boldsymbol{b}^{H} \boldsymbol{A}_{2} \boldsymbol{b}-2 \times R \boldsymbol{C}_{2}{ }^{H} \boldsymbol{b}+D_{2} \leq \\
& \boldsymbol{b}^{H} \boldsymbol{A}_{3} \boldsymbol{b}-2 \times R \boldsymbol{C}_{3}{ }^{H} \boldsymbol{b}+D_{3} \leq(
\end{aligned}
$$

where the corresponding parameters are defined as

$$
\begin{gathered}
\boldsymbol{b}=\operatorname{vec}(\boldsymbol{B}) \\
\boldsymbol{A}_{1}=\left(\boldsymbol{I} \otimes \overline{\boldsymbol{H}}^{H} \boldsymbol{F}^{H} \overline{\boldsymbol{G}}^{H} \boldsymbol{Q}^{H} \boldsymbol{Q} \overline{\boldsymbol{G}} \boldsymbol{F} \overline{\boldsymbol{H}}\right) \\
+\sigma_{H}^{2} \operatorname{tr}\left(\boldsymbol{F}^{H} \overline{\boldsymbol{G}}^{H} \boldsymbol{Q}^{H} \boldsymbol{Q} \overline{\boldsymbol{G}} \boldsymbol{F} \boldsymbol{\Sigma}_{H}\right)\left(\boldsymbol{I} \otimes \boldsymbol{\Psi}_{H}\right) \\
+\sigma_{G}^{2} \operatorname{tr}\left(\boldsymbol{Q}^{H} \boldsymbol{Q} \Sigma_{G}\right)\left(\boldsymbol{I} \otimes \overline{\boldsymbol{H}}^{H} \boldsymbol{F}^{H} \boldsymbol{\Psi}_{G} \boldsymbol{F} \overline{\boldsymbol{H}}\right) \\
\boldsymbol{A}_{2}=(\boldsymbol{I} \otimes \boldsymbol{I}) \\
\boldsymbol{A}_{3}=\left(\boldsymbol{I} \otimes \overline{\boldsymbol{H}}^{H} \boldsymbol{F}^{H} \boldsymbol{F} \overline{\boldsymbol{H}}\right)+\sigma_{H}^{2} \operatorname{tr}\left(\boldsymbol{F}^{H} \boldsymbol{F} \Sigma_{\boldsymbol{H}}\right)\left(\boldsymbol{I} \otimes \boldsymbol{\Psi}_{H}\right) \\
\boldsymbol{C}_{\boldsymbol{1}}=\operatorname{vec}\left[(\boldsymbol{Q} \overline{\boldsymbol{G}} \boldsymbol{F} \overline{\boldsymbol{H}})^{H}\right] \\
\boldsymbol{C}_{2}=\boldsymbol{0} \\
\boldsymbol{C}_{3}=\boldsymbol{0} \\
D_{1}=0 \\
D_{2}=-P_{s} \\
D_{3}=-P_{r}+\operatorname{tr}\left(\boldsymbol{F} \boldsymbol{R}_{w} \boldsymbol{F}^{H}\right)
\end{gathered}
$$

By introducing an auxiliary variable $\mathrm{t}$, the problem can be converted into the following semidefinite programming (SDP) problem [12]-[13].

$$
\begin{aligned}
\min _{\boldsymbol{B}, \mathrm{t}} t & \left.\begin{array}{cc}
\boldsymbol{I} & \boldsymbol{A}_{1}^{1 / 2} \boldsymbol{b} \\
\left(\boldsymbol{A}_{1}^{1 / 2} \boldsymbol{b}\right)^{H} & t+2 \times R\left(\boldsymbol{C}_{\boldsymbol{1}}{ }^{H} \boldsymbol{b}\right)-D_{\boldsymbol{1}}
\end{array}\right] \geq \boldsymbol{0} \\
& {\left[\begin{array}{cc}
\boldsymbol{I} & \boldsymbol{A}_{i}^{1 / 2} \boldsymbol{b} \\
\left(\boldsymbol{A}_{i}^{1 / 2} \boldsymbol{b}\right)^{H} & 2 \times R\left(\boldsymbol{C}_{i}{ }^{H} \boldsymbol{b}\right)-D_{i}
\end{array}\right] \geq \boldsymbol{0}, i=2,3 }
\end{aligned}
$$

which can be efficiently solved by the interior-point method. In particular, the problem(22) can be solved by the CVX MATLAB toolbox for disciplined convex programming [14].

\section{B. Optimization of Relay Precoding matrix $\boldsymbol{F}$}

The sub-problem to optimize $\boldsymbol{F}$ for fixed $\boldsymbol{B}$ and $\boldsymbol{Q}$ can be written as

$$
\begin{aligned}
& \arg \min _{\boldsymbol{F}}\{M S E\} \\
& \text { s.t } \operatorname{tr}\left[\boldsymbol{F} \mathbf{Z} \boldsymbol{F}^{H}\right] \leq P_{r}
\end{aligned}
$$

It can be easily proved that the problem (23) is convex ${ }^{[15]}$ Therefore $\boldsymbol{F}$ can be calculated from the Karush-Kuhn-Tucker (KKT) conditions. The Lagrangian function of (23) is formulated as

$$
L(\boldsymbol{F}, \lambda)=\operatorname{MSE}(\boldsymbol{B}, \boldsymbol{F}, \boldsymbol{Q})+\lambda\left(\operatorname{tr}\left[\boldsymbol{F Z} \boldsymbol{F}^{H}\right]-P_{r}\right)
$$

where $\lambda$ is the Lagrange multiplier. By taking the derivative of $L(\boldsymbol{F}, \lambda)$ with respect to $\boldsymbol{F}^{*}$, together with the complementary slackness and the relay station transmit power constraint, the KKT conditions can be obtained as

$$
\begin{gathered}
\left.\frac{\partial L(\boldsymbol{F}, \lambda)}{\partial \overline{\boldsymbol{F}}}\right|_{\overline{\boldsymbol{F}}=\boldsymbol{F}^{*}}=0 \\
\operatorname{tr}\left[\boldsymbol{F} \boldsymbol{Z} \boldsymbol{F}^{H}\right]-P_{r}<0 \\
\lambda>0 \\
\lambda\left(\operatorname{tr}\left[\boldsymbol{F} \boldsymbol{Z} \boldsymbol{F}^{H}\right]-P_{r}\right)=0
\end{gathered}
$$

The relay precoding matrix $\boldsymbol{F}$ is calculated as

$$
\begin{aligned}
\boldsymbol{F}= & \left(\overline{\boldsymbol{G}}^{H} \boldsymbol{Q}^{H} \boldsymbol{Q} \overline{\boldsymbol{G}}+\sigma_{G}^{2} \operatorname{tr}\left(\boldsymbol{Q}^{H} \boldsymbol{Q} \boldsymbol{\Sigma}_{G}\right) \boldsymbol{\Psi}_{G}+\lambda \boldsymbol{I}_{n_{r}}\right)^{+} \\
& \overline{\boldsymbol{G}}^{H} \boldsymbol{Q}^{H} \boldsymbol{B}^{H} \overline{\boldsymbol{H}}^{H} \boldsymbol{Z}^{+}
\end{aligned}
$$

where the optimal $\lambda$ should be chosen to satisfy (27)and (28), it is shown that $\lambda$ is bounded as $0 \leq \lambda \leq \sqrt{\frac{\overline{\boldsymbol{G}}^{H} \boldsymbol{Q}^{H} \boldsymbol{B}^{H} \overline{\boldsymbol{H}}^{H} \boldsymbol{Z}^{+} \overline{\boldsymbol{H}} \boldsymbol{B} \boldsymbol{Q} \overline{\boldsymbol{G}}}{P_{r}}}$, then the optimal $\lambda$ can be conveniently found using the bisection search method ${ }^{[12]}$.

To simplify the original problem, we introduce a linear scaling $\eta>0$ in the equalizer, and replace $\boldsymbol{Q}$ with a scaled version $\eta^{-1} \boldsymbol{Q}$, after some manipulation ${ }^{[12]}$, we can get

$$
\begin{aligned}
\boldsymbol{F}= & \eta\left(\overline{\boldsymbol{G}}^{H} \boldsymbol{Q}^{H} \boldsymbol{Q} \overline{\boldsymbol{G}}+\sigma_{G}^{2} \operatorname{tr}\left(\boldsymbol{Q}^{H} \boldsymbol{Q} \boldsymbol{\Sigma}_{G}\right) \boldsymbol{\Psi}_{G}+\theta \boldsymbol{I}_{n_{r}}\right)^{+} \\
& \overline{\boldsymbol{G}}^{H} \boldsymbol{Q}^{H} \boldsymbol{B}^{H} \overline{\boldsymbol{H}}^{H} \boldsymbol{Z}^{+}
\end{aligned}
$$

where

$$
\begin{gathered}
\theta=\frac{\operatorname{tr}\left(\boldsymbol{Q R}_{n} \boldsymbol{Q}^{H}\right)}{P_{r}} \\
M S E_{\min }(\boldsymbol{B}, \boldsymbol{Q})= \\
\operatorname{tr}\left(\boldsymbol{I}_{n_{s}}\right)-\operatorname{tr}\left[\boldsymbol{B}^{H} \overline{\boldsymbol{H}}^{H} \boldsymbol{Z}^{+} \overline{\boldsymbol{H}} \boldsymbol{B} \boldsymbol{Q} \overline{\boldsymbol{G}}\right. \\
\left.\left(\overline{\boldsymbol{G}}^{H} \boldsymbol{Q}^{H} \boldsymbol{Q} \overline{\boldsymbol{G}}+\sigma_{G}^{2} \operatorname{tr}\left(\boldsymbol{Q}^{H} \boldsymbol{Q} \Sigma_{G}\right) \boldsymbol{\Psi}_{G}+\theta \boldsymbol{I}_{n_{r}}\right)^{+} \overline{\boldsymbol{G}}^{H} \boldsymbol{Q}^{H}\right]
\end{gathered}
$$

Proof: See Appendix A.

C. Optimization of Equalizer Matrix $\boldsymbol{Q}$

The objective is to minimize

$$
\begin{aligned}
\operatorname{MSE}(\boldsymbol{Q})= & \operatorname{tr}\left(\boldsymbol{I}_{n_{s}}\right)-\operatorname{tr}\left[\boldsymbol{B}^{H} \overline{\boldsymbol{H}}^{H} \boldsymbol{Z}^{+} \overline{\boldsymbol{H}} \boldsymbol{B} \boldsymbol{Q} \overline{\boldsymbol{G}}\right. \\
& \left.\left(\overline{\boldsymbol{G}}^{H} \boldsymbol{Q}^{H} \boldsymbol{Q} \overline{\boldsymbol{G}}+\sigma_{G}^{2} \operatorname{tr}\left(\boldsymbol{Q}^{H} \boldsymbol{Q} \Sigma_{G}\right) \boldsymbol{\Psi}_{G}+\theta \boldsymbol{I}_{n_{r}}\right)^{+} \overline{\boldsymbol{G}}^{H} \boldsymbol{Q}^{H}\right] \\
= & \operatorname{tr}\left(\boldsymbol{E}_{H}\right)+\operatorname{tr}\left(\boldsymbol{E}_{G}\right)-\operatorname{tr}\left[\boldsymbol{E}_{H} \boldsymbol{E}_{G}\right]
\end{aligned}
$$

where

$$
\begin{aligned}
& \boldsymbol{E}_{H}=\boldsymbol{I}_{n_{s}}-\boldsymbol{B}^{H} \overline{\boldsymbol{H}}^{H} \boldsymbol{Z}^{+} \overline{\boldsymbol{H}} \boldsymbol{B} \\
& \boldsymbol{\beta}_{n}=\sigma_{G}^{2} \operatorname{tr}\left(\boldsymbol{Q}^{H} \boldsymbol{Q} \Sigma_{G}\right) \boldsymbol{\Psi}_{G}+\theta \boldsymbol{I}_{n_{r}} \\
&=\sigma_{G}^{2} \operatorname{tr}\left(\boldsymbol{Q}^{H} \boldsymbol{Q} \Sigma_{G}\right) \boldsymbol{\Psi}_{G}+\theta \boldsymbol{I}_{n_{r}} \\
& \leq \sigma_{G}^{2} \operatorname{tr}\left(\boldsymbol{Q}^{H} \boldsymbol{Q}\right) \lambda_{\max }\left(\Sigma_{G}\right) \boldsymbol{\Psi}_{G}+\theta \boldsymbol{I}_{n_{r}} \\
&=\left(\sigma_{G}^{2} \boldsymbol{P}_{r} \lambda_{\max }\left(\Sigma_{G}\right) \boldsymbol{R}_{n}{ }^{-1} \boldsymbol{\Psi}_{G}+\boldsymbol{I}_{n_{r}}\right) \theta_{n} \\
& \boldsymbol{E}_{G}=\left(\boldsymbol{I}_{n_{s}}+\boldsymbol{Q}_{n} \overline{\boldsymbol{G}} \boldsymbol{\beta}_{n}^{-1} \overline{\boldsymbol{G}}^{H} \boldsymbol{Q}_{n}{ }^{H}\right)^{-1}
\end{aligned}
$$


The unconstrained optimization problem can be solved by a gradient-based line search algorithm [9].

$$
\begin{aligned}
\Delta \boldsymbol{Q}_{n} & =-\nabla_{Q^{*}} \boldsymbol{M S E}(\boldsymbol{Q}) \\
& =-\eta_{n}^{-2} \boldsymbol{Q} \boldsymbol{R}_{n}+\boldsymbol{E}_{G} \boldsymbol{B}^{H} \overline{\boldsymbol{H}}^{H} \boldsymbol{Z}^{+} \overline{\boldsymbol{H}} \boldsymbol{B} \boldsymbol{E}_{G} \boldsymbol{Q} \overline{\boldsymbol{G}} \boldsymbol{\beta}_{n}^{-1} \overline{\boldsymbol{G}}^{H}
\end{aligned}
$$

This method is summarized in the following Table I.

TABLE I: GRADIENT DESCENT ALGORITHM

$$
\begin{aligned}
& \text { 1.Choose } P_{r} \text { and } \varepsilon>0 \\
& \text { 2.Choose line search parameters: } \\
& \qquad \rho \in(0.1,0.8), c \in(0,0.5), \bar{\alpha}, K_{\max } \\
& \text { 3.Initialize the counter } n=-1 \text { and the equalizer } \boldsymbol{Q}_{0}=\boldsymbol{I} \\
& \text { 4.Repeat } \\
& \text { 5.Increment counter } n \leftarrow n+1 \\
& \text { 6.Compute: } \Delta Q_{n} \text { from }(37) \\
& \text { 7.Set } \alpha \leftarrow \bar{\alpha} \\
& \text { 8. } \quad \operatorname{Repeat}^{\text {9. }} \quad \alpha \leftarrow \rho \alpha \\
& \text { 10. Until } \quad \mathbf{M S E}_{\min }\left(\boldsymbol{Q}_{n}+\alpha \Delta \boldsymbol{Q}_{n}\right) \\
& \quad \leq E_{\min }\left(\boldsymbol{Q}_{n}\right)-c \alpha\left\|\Delta \boldsymbol{Q}_{n}\right\|_{F}^{2} \text { or } n>K_{\max } \\
& \text { 11.Update } \boldsymbol{Q}_{n+1} \leftarrow \boldsymbol{Q}_{n}+\alpha \Delta \boldsymbol{Q}_{n} \\
& \text { 12.Until }\left\|\Delta \boldsymbol{Q}_{n}\right\|_{F}^{2}<\varepsilon
\end{aligned}
$$

\section{Joint Design}

Since optimization of the precoder $\boldsymbol{B}, \boldsymbol{F}$ and $\boldsymbol{Q}$ have been discussed. An alternating algorithm for the joint design of these three matrices was proposed. This framework is described in Table II.

TABLE II: JOINT DESIGN ALGORITHM

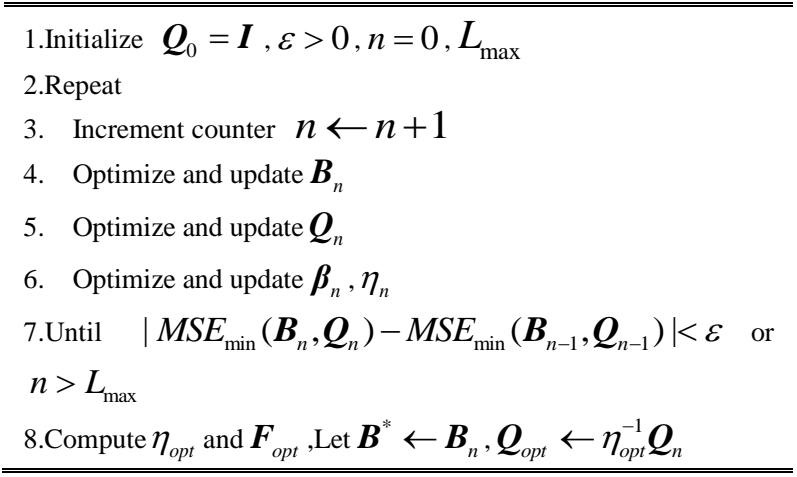

\section{Simulation RESUlT}

In this section, simulation results are provided to evaluate the performance of the proposed approaches in a flat Rayleigh fading environment. The source node transmits independent uncoded QPSK symbol streams to the corresponding receiver. The antenna correlation matrices are generated using the exponential model. The elements of $\Sigma_{H}$ are given by $\Sigma_{H}(\mathrm{~m}, \mathrm{n})=\rho^{|\mathrm{m}-\mathrm{n}|}, 1 \leq m, n \leq n_{r}$, where $\rho$ denotes the correlation coefficients. Moreover, $\boldsymbol{\Psi}_{H}, \Sigma_{G}$ and $\boldsymbol{\Psi}_{G}$ can be obtained similarly, and the same correlation coefficients are used. The CSI error variances are given by $\sigma_{H}^{2}=\sigma_{G}^{2}=\sigma_{e}^{2}$, and the average signal-to-noise ratios (SNRs) for two hops are defined as $S N R_{s r}=P_{s} /\left(\mathrm{n}_{s} \sigma_{w}^{2}\right)$ and $S N R_{r d}=P_{r} /\left(\mathrm{n}_{r} \sigma_{n}^{2}\right)$, respectively. The following parameters are chosen throughout this section: $\sigma_{H}^{2}=\sigma_{G}^{2}=\sigma_{e}^{2}=0.005, \rho=0.4, n_{s}=n_{r}=n_{d}=4$.

To illustrate the advantages of the proposed alternating approach, some other approaches are also included for comparison. The methods under comparison are:

- Joint design $\boldsymbol{B}$ and $\boldsymbol{F}: \boldsymbol{Q} \propto \boldsymbol{I}$.

- Joint design $\boldsymbol{F}$ and $\boldsymbol{Q}: \boldsymbol{B} \propto \boldsymbol{I}$.

- $\quad$ Proposed Approach:Joint design of $\boldsymbol{B}, \boldsymbol{F}$ and $\boldsymbol{Q}$.

The average BERs are shown in Fig. 2 and Fig. 3, where we let $S N R_{s r}=20 \mathrm{~d} B$ or $S N R_{r d}=20 d B$. As expected, Joint design $\boldsymbol{F}$ and $\boldsymbol{Q}$ outperforms Joint design $\boldsymbol{F}$ and $\boldsymbol{Q}$ at low-to-mid SNRs, The joint design $\boldsymbol{B}, \boldsymbol{F}$ and $\boldsymbol{Q}$ is significantly better than other approaches at mid-to-high SNRs and enable an additional SNR gain of $2-4 \mathrm{~dB}$, which could demonstrate the effectiveness of the proposed approach.

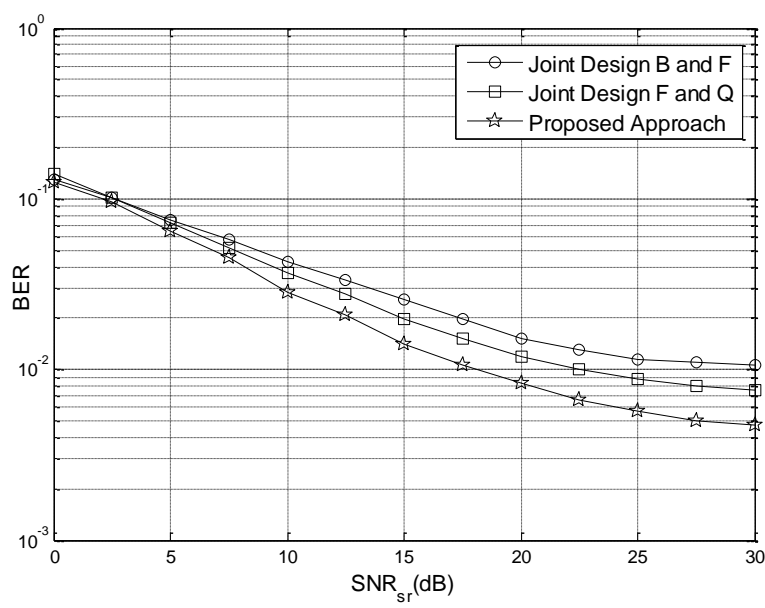

Fig. 2. BER versus $S N R_{s r}$ with $S N R_{r d}=20 \mathrm{~dB}$

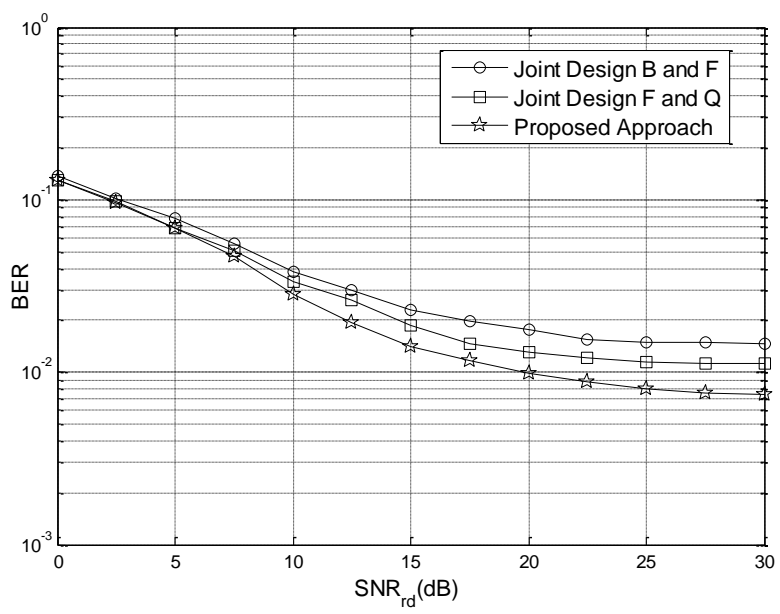

Fig. 3. BER versus $S N R_{r d}$ with $S N R_{s r}=20 \mathrm{~dB}$

\section{CONCLUSION}

In this paper, by incorporating the antenna correlations and channel estimation errors, the joint transceiver design based on the minimum sum MSE criterion for AF-MIMO 
relay systems is investigated. We decouple the original problem into three convex sub-problems firstly and then propose an iterative algorithm with guaranteed convergence. Subsequently, we derive the certain precoding and equalizer matrices by employing the interior-point method, the Lagrange multiplier method and the Gradient Descent method. The performance advantage of the proposed approach is demonstrated via simulation results.

\section{ACKNOWLEDGMENT}

This work is supported by the Fundamental Research Funds for the Central Universities. ( NO.NS2015046, NO.NS2016044).

\section{APPENDIX A}

For any $\eta^{-1} \boldsymbol{Q}$ with $\eta>0$, the optimal relaying matrix is obtained by replacing $\boldsymbol{Q}$ with $\eta^{-1} \boldsymbol{Q}$ in (29). Henceforth, $\boldsymbol{F}$ can be calculated as:

$$
\begin{aligned}
\boldsymbol{F}= & \eta\left(\overline{\boldsymbol{G}}^{H} \boldsymbol{Q}^{H} \boldsymbol{Q} \overline{\boldsymbol{G}}+\sigma_{G}^{2} \operatorname{tr}\left(\boldsymbol{Q}^{H} \boldsymbol{Q} \boldsymbol{\Sigma}_{G}\right) \boldsymbol{\Psi}_{G}+\lambda \eta^{2} \boldsymbol{I}_{n_{r}}\right)^{+} \\
& \overline{\boldsymbol{G}}^{H} \boldsymbol{Q}^{H} \boldsymbol{B}^{H} \overline{\boldsymbol{H}}^{H} \boldsymbol{Z}^{+}
\end{aligned}
$$

The corresponding Lagrangian function of (23) is given by

$$
\begin{aligned}
& \operatorname{MSE}(\boldsymbol{F}, \lambda)=\operatorname{MSE}(\boldsymbol{B}, \boldsymbol{F}, \boldsymbol{Q})+\lambda\left(\operatorname{tr}\left[\boldsymbol{F Z} \boldsymbol{F}^{H}\right]-P_{r}\right) \\
& =\operatorname{tr}\left(\boldsymbol{I}_{n_{s}}\right)-\operatorname{tr}(\boldsymbol{Q} \overline{\boldsymbol{G}} \boldsymbol{F} \overline{\boldsymbol{H}} \boldsymbol{B})+\operatorname{tr}\left(\boldsymbol{Q} \boldsymbol{R}_{n} \boldsymbol{Q}^{H}\right)-\lambda P_{r} \\
& =\operatorname{tr}\left(\boldsymbol{I}_{n_{s}}\right)-\eta^{-1} \operatorname{tr}(\boldsymbol{Q} \overline{\boldsymbol{G}} \boldsymbol{F} \overline{\boldsymbol{H}} \boldsymbol{B})+\eta^{-2} \operatorname{tr}\left(\boldsymbol{Q} \boldsymbol{R}_{n} \boldsymbol{Q}^{H}\right)-\lambda P_{r}
\end{aligned}
$$

Taking (26) and (31) into(39), we can get

$$
\begin{aligned}
\operatorname{MSE}(\boldsymbol{F}, \lambda)= & \operatorname{tr}\left(\boldsymbol{I}_{n_{s}}\right)-\eta^{-1} \operatorname{tr}(\boldsymbol{Q} \overline{\boldsymbol{G}} \boldsymbol{F} \overline{\boldsymbol{H}} \boldsymbol{B}) \\
& +\eta^{-2} \operatorname{tr}\left(\boldsymbol{Q} \boldsymbol{R}_{n} \boldsymbol{Q}^{H}\right)-\lambda \operatorname{tr}\left[\boldsymbol{F Z} \boldsymbol{F}^{H}\right] \\
= & \operatorname{tr}\left(\boldsymbol{I}_{n_{s}}\right)-\eta^{-1} \operatorname{tr}(\boldsymbol{Q} \overline{\boldsymbol{G}} \boldsymbol{F} \overline{\boldsymbol{H}} \boldsymbol{B})+\eta^{-2} \theta P_{r}-\lambda \operatorname{tr}\left[\boldsymbol{F} \boldsymbol{Z F}^{H}\right](40) \\
= & \operatorname{tr}\left(\boldsymbol{I}_{n_{s}}\right)-\eta^{-1} \operatorname{tr}(\boldsymbol{Q} \overline{\boldsymbol{G}} \boldsymbol{F} \overline{\boldsymbol{H}} \boldsymbol{B})+\left(\eta^{-2} \theta-\lambda\right) \operatorname{tr}\left[\boldsymbol{F} \boldsymbol{Z} \boldsymbol{F}^{H}\right] \\
= & \operatorname{tr}\left(\boldsymbol{I}_{n_{s}}\right)-\operatorname{tr}\left\{\eta^{-1} \boldsymbol{Q} \overline{\boldsymbol{G}} \boldsymbol{F} \overline{\boldsymbol{H}} \boldsymbol{B}-\left(\eta^{-2} \theta-\lambda\right) \boldsymbol{F} \mathbf{Z} \boldsymbol{F}^{H}\right\}
\end{aligned}
$$

where

$$
\begin{aligned}
& \eta^{-1} \boldsymbol{Q} \overline{\boldsymbol{G}} \boldsymbol{F} \overline{\boldsymbol{H}} \boldsymbol{B}=\boldsymbol{Q} \overline{\boldsymbol{G}}\left(\overline{\boldsymbol{G}}^{H} \boldsymbol{Q}^{H} \boldsymbol{Q} \overline{\boldsymbol{G}}+\sigma_{G}^{2} \operatorname{tr}\left(\boldsymbol{Q}^{H} \boldsymbol{Q} \boldsymbol{\Sigma}_{\boldsymbol{G}}\right) \boldsymbol{\Psi}_{\boldsymbol{G}}+\lambda \eta^{2} \boldsymbol{I}_{n_{r}}\right)^{+} \\
& \overline{\boldsymbol{G}}^{H} \boldsymbol{Q}^{H} \boldsymbol{B}^{H} \overline{\boldsymbol{H}}^{H} \boldsymbol{Z}^{+} \overline{\boldsymbol{H}} \boldsymbol{B}
\end{aligned}
$$

$$
\begin{aligned}
\left(\eta^{-2} \theta-\lambda\right) \boldsymbol{F} \boldsymbol{Z} \boldsymbol{F}^{H} \\
=\left(\eta^{-2} \theta-\lambda\right) \eta^{2}\left(\overline{\boldsymbol{G}}^{H} \boldsymbol{Q}^{H} \boldsymbol{Q} \overline{\boldsymbol{G}}+\sigma_{G}^{2} \operatorname{tr}\left(\boldsymbol{Q}^{H} \boldsymbol{Q} \Sigma_{G}\right) \boldsymbol{\Psi}_{G}+\lambda \eta^{2} \boldsymbol{I}_{n_{r}}\right)^{+} \\
\overline{\boldsymbol{G}}^{H} \boldsymbol{Q}^{H} \boldsymbol{B}^{H} \overline{\boldsymbol{H}}^{H} \boldsymbol{Z}^{+} \overline{\boldsymbol{H}} \boldsymbol{B} \boldsymbol{Q} \overline{\boldsymbol{G}} \\
\left(\overline{\boldsymbol{G}}^{H} \boldsymbol{Q}^{H} \boldsymbol{Q} \overline{\boldsymbol{G}}+\sigma_{G}^{2} \operatorname{tr}\left(\boldsymbol{Q}^{H} \boldsymbol{Q} \boldsymbol{\Sigma}_{\boldsymbol{G}}\right) \boldsymbol{\Psi}_{G}+\lambda \eta^{2} \boldsymbol{I}_{n_{r}}\right)^{-H} \\
=\left(\theta-\lambda \eta^{2}\right)\left(\overline{\boldsymbol{G}}^{H} \boldsymbol{Q}^{H} \boldsymbol{Q} \overline{\boldsymbol{G}}+\sigma_{G}^{2} \operatorname{tr}\left(\boldsymbol{Q}^{H} \boldsymbol{Q} \Sigma_{\boldsymbol{G}}\right) \boldsymbol{\Psi}_{\boldsymbol{G}}+\lambda \eta^{2} \boldsymbol{I}_{n_{r}}\right)^{-2} \\
\overline{\boldsymbol{G}}^{H} \boldsymbol{Q}^{H} \boldsymbol{B}^{H} \overline{\boldsymbol{H}}^{H} \boldsymbol{Z}^{+} \overline{\boldsymbol{H}} \boldsymbol{B} \boldsymbol{Q} \overline{\boldsymbol{G}} \\
=\left(\theta-\lambda \eta^{2}\right) \boldsymbol{Q} \overline{\boldsymbol{G}}\left(\overline{\boldsymbol{G}}^{H} \boldsymbol{Q}^{H} \boldsymbol{Q} \overline{\boldsymbol{G}}+\sigma_{G}^{2} \operatorname{tr}\left(\boldsymbol{Q}^{H} \boldsymbol{Q} \Sigma_{\boldsymbol{G}}\right) \boldsymbol{\Psi}_{\boldsymbol{G}}+\lambda \eta^{2} \boldsymbol{I}_{n_{r}}\right)^{-2} \\
\overline{\boldsymbol{G}}^{H} \boldsymbol{Q}^{H} \boldsymbol{B}^{H} \overline{\boldsymbol{H}}^{H} \boldsymbol{Z}^{+} \overline{\boldsymbol{H} \boldsymbol{B}}
\end{aligned}
$$

Then

$$
\begin{aligned}
& \operatorname{tr}\left\{\boldsymbol{Q} \overline{\boldsymbol{G}} \boldsymbol{F} \overline{\boldsymbol{H}} \boldsymbol{B}-\left(\theta-\lambda \eta^{2}\right) \boldsymbol{F} \mathbf{Z} \boldsymbol{F}^{H}\right\} \\
& =\boldsymbol{Q} \overline{\boldsymbol{G}}\left(\overline{\boldsymbol{G}}^{H} \boldsymbol{Q}^{H} \boldsymbol{Q} \overline{\boldsymbol{G}}+\sigma_{G}^{2} \operatorname{tr}\left(\boldsymbol{Q}^{H} \boldsymbol{Q} \boldsymbol{\Sigma}_{\boldsymbol{G}}\right) \boldsymbol{\Psi}_{\boldsymbol{G}}+\lambda \eta^{2} \boldsymbol{I}_{n_{r}}\right)^{+} \\
& \overline{\boldsymbol{G}}^{H} \boldsymbol{Q}^{H} \boldsymbol{B}^{H} \overline{\boldsymbol{H}}^{H} \boldsymbol{Z}^{+} \overline{\boldsymbol{H}} \boldsymbol{B} \\
& -\left(\theta-\lambda \eta^{2}\right) \boldsymbol{Q} \overline{\boldsymbol{G}}\left(\overline{\boldsymbol{G}}^{H} \boldsymbol{Q}^{H} \boldsymbol{Q} \overline{\boldsymbol{G}}+\sigma_{G}^{2} \operatorname{tr}\left(\boldsymbol{Q}^{H} \boldsymbol{Q} \boldsymbol{\Sigma}_{\boldsymbol{G}}\right) \boldsymbol{\Psi}_{\boldsymbol{G}}+\lambda \eta^{2} \boldsymbol{I}_{n_{r}}\right)^{-2} \\
& \overline{\boldsymbol{G}}^{H} \boldsymbol{Q}^{H} \boldsymbol{B}^{H} \overline{\boldsymbol{H}}^{H} \boldsymbol{Z}^{+} \overline{\boldsymbol{H}} \boldsymbol{B} \\
& =\boldsymbol{Q} \overline{\boldsymbol{G}}\left(\overline{\boldsymbol{G}}^{H} \boldsymbol{Q}^{H} \boldsymbol{Q} \overline{\boldsymbol{G}}+\sigma_{G}^{2} \operatorname{tr}\left(\boldsymbol{Q}^{H} \boldsymbol{Q} \boldsymbol{\Sigma}_{G}\right) \boldsymbol{\Psi}_{G}+\lambda \eta^{2} \boldsymbol{I}_{n_{r}}\right)^{-2} \\
& \left(\overline{\boldsymbol{G}}^{H} \boldsymbol{Q}^{H} \boldsymbol{Q} \overline{\boldsymbol{G}}+\sigma_{G}^{2} \operatorname{tr}\left(\boldsymbol{Q}^{H} \boldsymbol{Q} \boldsymbol{\Sigma}_{G}\right) \boldsymbol{\Psi}_{G}+\lambda \eta^{2} \boldsymbol{I}_{n_{r}}-\left(\theta-\lambda \eta^{2}\right) \boldsymbol{I}_{n_{r}}\right) \\
& \overline{\boldsymbol{G}}^{H} \boldsymbol{Q}^{H} \boldsymbol{B}^{H} \overline{\boldsymbol{H}}^{H} \boldsymbol{Z}^{+} \overline{\boldsymbol{H}} \boldsymbol{B} \\
& =\boldsymbol{Q} \overline{\boldsymbol{G}}\left(\overline{\boldsymbol{G}}^{H} \boldsymbol{Q}^{H} \boldsymbol{Q} \overline{\boldsymbol{G}}+\sigma_{G}^{2} \operatorname{tr}\left(\boldsymbol{Q}^{H} \boldsymbol{Q} \Sigma_{G}\right) \boldsymbol{\Psi}_{G}+\lambda \eta^{2} \boldsymbol{I}_{n_{r}}\right)^{-2} \\
& \left(\overline{\boldsymbol{G}}^{H} \boldsymbol{Q}^{H} \boldsymbol{Q} \overline{\boldsymbol{G}}+\sigma_{G}^{2} \operatorname{tr}\left(\boldsymbol{Q}^{H} \boldsymbol{Q} \Sigma_{G}\right) \boldsymbol{\Psi}_{G}+2 \lambda \eta^{2} \boldsymbol{I}_{n_{r}}-\theta \boldsymbol{I}_{n_{r}}\right) \\
& \overline{\boldsymbol{G}}^{H} \boldsymbol{Q}^{H} \boldsymbol{B}^{H} \overline{\boldsymbol{H}}^{H} \boldsymbol{Z}^{+} \overline{\boldsymbol{H}} \boldsymbol{B}
\end{aligned}
$$

So the equation (40) becomes

$$
\operatorname{MSE}(\boldsymbol{F}, \lambda)=\operatorname{tr}\left(\boldsymbol{I}_{n_{s}}\right)-g(\xi)
$$

where

$$
\begin{aligned}
g(\xi)= & \operatorname{tr}\left\{\boldsymbol{Q} \overline{\boldsymbol{G}}\left(\overline{\boldsymbol{G}}^{H} \boldsymbol{Q}^{H} \boldsymbol{Q} \overline{\boldsymbol{G}}+\sigma_{G}^{2} \operatorname{tr}\left(\boldsymbol{Q}^{H} \boldsymbol{Q} \Sigma_{\boldsymbol{G}}\right) \boldsymbol{\Psi}_{G}+\xi \boldsymbol{I}_{n_{r}}\right)^{-2}\right. \\
& \left(\overline{\boldsymbol{G}}^{H} \boldsymbol{Q}^{H} \boldsymbol{Q} \overline{\boldsymbol{G}}+\sigma_{G}^{2} \operatorname{tr}\left(\boldsymbol{Q}^{H} \boldsymbol{Q} \Sigma_{\boldsymbol{G}}\right) \boldsymbol{\Psi}_{G}+2 \xi \boldsymbol{I}_{n_{r}}-\theta \boldsymbol{I}_{n_{r}}\right)(45) \\
& \left.\overline{\boldsymbol{G}}^{H} \boldsymbol{Q}^{H} \boldsymbol{B}^{H} \overline{\boldsymbol{H}}^{H} \boldsymbol{Z}^{+} \overline{\boldsymbol{H}} \boldsymbol{B}\right\}
\end{aligned}
$$

Define the SVD

$$
\overline{\boldsymbol{G}}^{H} \boldsymbol{Q}^{H} \boldsymbol{Q} \overline{\boldsymbol{G}}+\sigma_{G}^{2} \operatorname{tr}\left(\boldsymbol{Q}^{H} \boldsymbol{Q} \boldsymbol{\Sigma}_{\boldsymbol{G}}\right) \boldsymbol{\Psi}_{\boldsymbol{G}}=\boldsymbol{U} \boldsymbol{M} \boldsymbol{U}^{H}
$$

The problem is then reduced to that of maximizing

$$
\begin{array}{r}
g(\xi)=\operatorname{tr}\left\{\left(\mathbf{M}+\xi \boldsymbol{I}_{n_{r}}\right)^{-2}\left(\boldsymbol{M}+2 \xi \boldsymbol{I}_{n_{r}}-\theta \boldsymbol{I}_{n_{r}}\right)\right. \\
\left.\boldsymbol{U}^{H} \overline{\boldsymbol{G}}^{H} \boldsymbol{Q}^{H} \boldsymbol{B}^{H} \overline{\boldsymbol{H}}^{H} \boldsymbol{Z}^{+} \overline{\boldsymbol{H}} \boldsymbol{B} \boldsymbol{Q} \overline{\boldsymbol{G}} \boldsymbol{U}\right\}
\end{array}
$$

where $\quad \boldsymbol{\Lambda}=\boldsymbol{U}^{H} \overline{\boldsymbol{G}}^{H} \boldsymbol{Q}^{H} \boldsymbol{B}^{H} \overline{\boldsymbol{H}}^{H} \boldsymbol{Z}^{+} \overline{\boldsymbol{H}} \boldsymbol{B} \boldsymbol{Q} \overline{\boldsymbol{G}} \boldsymbol{U}$. Define $\alpha_{k} \geq 0$ as the kth diagonal entry of $\mathrm{M}$ and $\beta_{k} \geq 0$ as the(k, k)th entry of $\boldsymbol{\Lambda}$, we have

$$
g(\xi)=\sum_{k=1}^{n_{s}} \beta_{k} \frac{\alpha_{k}+2 \xi-\theta}{\left(\alpha_{k}+\xi\right)^{2}}
$$

whose first-order derivative

$$
\begin{aligned}
\frac{d g}{d \xi}=\sum_{k=1}^{n_{s}} \beta_{k} \frac{2\left(\alpha_{k}+\xi\right)^{2}-2\left(\alpha_{k}+2 \xi-\theta\right)\left(\alpha_{k}+\xi\right)}{\left(\alpha_{k}+\xi\right)^{4}} \\
=\sum_{k=1}^{n_{s}} \beta_{k} \frac{2\left(\alpha_{k}+\xi\right)-2\left(\alpha_{k}+2 \xi-\theta\right)}{\left(\alpha_{k}+\xi\right)^{4}} \\
=-\sum_{k=1}^{n_{s}} 2 \beta_{k} \frac{\theta-\xi}{\left(\alpha_{k}+\xi\right)^{3}}=0
\end{aligned}
$$

Therefore $\xi=\lambda \eta^{2}=\theta$ is the unique solution to maximize $g(\xi)$ and $\eta$ should satisfy (26). Substituting $\lambda \eta^{2}=\theta$ into (38)and (39) leads to (30) and (32). 


\section{REFERENCES}

[1] I. E. Telatar, "Capacity of multi-antenna Gaussian channels," European Transactions Telecommunication, U.K.: Wiley, 1999, pp. 585-595.

[2] L. Sanguinetti, D. A. A. Amico, and Y. Rong, "A tutorial on the optimization of amplify-and-forward MIMO relay systems," IEEE Journal on Selected Areas in Communications, vol. 30, pp. 1331-1346, Sep 2012.

[3] H. Shen, W. Xu, and C. Zhao, "Robust transceiver for AF MIMO relaying with direct link: A globally optimal solution," IEEE Signal Processing Letters, vol. 21, pp. 947-951, Aug 2014.

[4] Y. Rong, X. Tang, and Y. Hua, "A unified framework for optimizing linear non-regenerative multicarrier MIMO relay communication systems," IEEE Trans on Signal Processing, vol. 57, pp. 4837-4851, Dec 2009.

[5] C. Song, K. J. Lee, and I. Lee, "MMSE based transceiver designs in closed-loop non-regenerative MIMO relaying systems," IEEE Trans, on Wireless Communications, vol. 9, pp. 2310-2319, July 2010.

[6] C. Zhao and B. Champagne, "A unified approach to optimal transceiver design for non-regenerative MIMO relaying," IEEE Trans, on Vehicular Technology, vol. 64, pp. 2938-2951, July 2015.

[7] J. Zou, W. Liu, H. W. Luo et al., "Linear precoder design for amplify-and-forward MIMO relay systems with channel estimation errors," Journal of Shanghai Jiaotong University, vol. 45, pp. 1050-1049, Feb 2011.

[8] L. Zhang, Y. Cai, M. Zhao et al., "Robust tomlinson-harashima precoding design in amplify-and-forward MIMO relay systems via MMSE criterion," in Proc. 2013 IEEE Wireless Communications and Networking Conference, pp. 2727-2732, Shanghai, 2013.

[9] C. Xing, S. Li, Z. Fei, and J. Kuang, "How to understand linear minimum mean-square-error transceiver design for multiple-input-multiple-output systems from quadratic matrix programming," IET Communications, vol. 7, pp. 1231-1242, Aug 2013.

[10] J. Wagner, B. Rankov, and A. Wittneben, "Large analysis of amplify-and-forward MIMO relay channels with correlated Rayleigh fading," IEEE Transactions on Information Theory, 2008, vol. 54, no. 12 , pp. $5735-5746$.

[11] R. H. Y. Louie, Y. Li, H. A. Suraweera et al., "Performance analysis of beamforming in two hop amplify and forward relay networks with antenna correlation," IEEE Transactions on Wireless Communications, vol. 8, pp. 3132-3141, June 2009.

[12] M. Ding and S D. Blostein, "MIMO minimum total MSE transceiver design with imperfect CSI at both ends," IEEE Trans on Signal Processing, vol. 57, pp. 1141-1150, March 2009.

[13] J. Liu, F. Gao, and Z. Qiu "Robust transceiver design for multi-user multiple-input multiple-output amplify-and-foward relay systems," IET Communications, vol. 8, pp. 2162-2170, March 2014.

[14] M. Grant and S. P. Boyd, "CVX: MATLAB software for disciplined convex programming," Global Optimization, 2014, pp. 155-210.

[15] S. Boyd and L. Vandenberghe, Convex Optimization, Cambridge University Press, 2004, pp. 156-180.

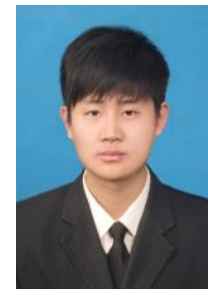

Junxu Su was born in Yueyang city, Hunan province, China, in 1993. He received the B.E. degree from Hebei University of Engineering, Handan, China, in 2014. He is now pursuing his B.S. degree of communication and information system in Nanjing University of Aeronautics and Astronautics. His research interests is precoding algorithm for MIMO relay system.

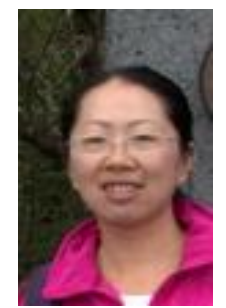

Xiaomin Chen was born in 1973. She received her BS in electronic engineering from Nanjing University of Aeronautics and Astronautics (NUAA), Nanjing, China, in 1997 and her MS and $\mathrm{PhD}$ in communication and information system from NUAA in 2001 and 2010, respectively. Since 2010, she has been an associate professor in the Department of Electronic Information Engineering, NUAA. Her research interests include adaptive optimization technique for next generation mobile communication, signal detection for MIMO system and MIMO relay system.

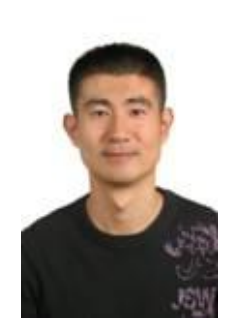

Qiuming Zhu was born in 1979. He received his BS in electronic engineering from Nanjing University of Aeronautics and Astronautics(NUAA), Nanjing, China, in 2002 and his $\mathrm{MS}$ and $\mathrm{PhD}$ in communication and information system from NUAA in 2005 and 2012, respectively. Since 2012, he has been an associate professor in the Department of Electronic Information Engineering, NUAA. His research interests include channel modeling for next generation mobile communication, wireless channel simulation and emulator, and MIMO relay system.

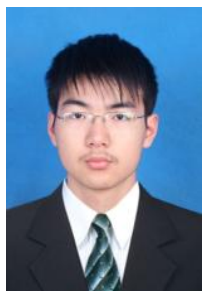

Yimin Zhu was born in 1990 . He received his B.E. degree from Nanjing University of Information Science and Technology, Nanjing, China, in 2013. And he received his BS in electronic engineering from Nanjing University of Aeronautics and Astronautics(NUAA), Nanjing, China. His research interests is power allocation and precoding algorithm for MIMO relay system.

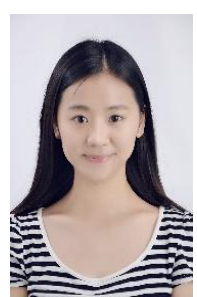

Zhu Fang was born in Anqing city, Anhui province, China, in 1993. She received the B.E. degree from Anqing Normal University in 2015. Currently, she is pursuing her M.S. degree of electronics and communication engineering in Nanjing University of Aeronautics and Astronautics. Her research interests include wireless communication technology, space-time coding and MIMO relay precoding and channel. 